\title{
Obtención de Pigmentos Inorgánicos para la Industria Cerámica por Síntesis Alternativa de Combustión en Solución
}

\author{
Manufacture of Inorganic Pigments for Ceramic Industry using the Alternative \\ Solution Combustion Synthesis. \\ Edgar Andrés Chavarriaga Miranda, Oscar Jaime Restrepo Baena \\ Facultad de Ciencias, Grupo de Investigación: Grupo del cemento y materiales de construcción, Universidad \\ Nacional de Colombia, Medellín, Colombia \\ eachavar@unal.edu.co \\ ojrestre@unal.edu.co
}

\begin{abstract}
Resumen - En este trabajo se presenta el proceso alternativo de obtención del pigmento inorgánico con estructura espinela $\mathrm{CuCr}_{2} \mathrm{O}_{4}$, por medio del método de síntesis conocido como combustión en solución, el cual consiste en una reacción de óxido reducción entre un combustible y un agente oxidante, también se obtuvo la estructura $\mathrm{CuCr}_{2} \mathrm{O}_{4}$ por el método de reacción de estado sólido, el cual emplea una temperatura de $1000{ }^{\circ} \mathrm{C}$ y un tiempo de proceso de $24 \mathrm{~h}$, en este caso también se presenta impurezas de $\mathrm{Cr}_{2} \mathrm{O}_{3}$. La estructura cristalina de los polvos sintetizados, se caracterizó por la técnica de difracción de rayos $X$ (DRX). La morfología de los polvos se determinó por microscopía electrónica de barrido (MEB). Finalmente, la espectrofotometría visible y la colorimetría CIE La*b* demostraron que los polvos son negros. Se concluye, que la obtención de $\mathrm{CuCr}_{2} \mathrm{O}_{4}$ por combustión en solución es energéticamente más eficiente que el método tradicional, ya que en el primer caso se emplearon como condiciones de proceso, una temperatura de $700{ }^{\circ} \mathrm{C}$ y un tiempo de $6 \mathrm{~h}$, lo cual mostró que la ruta de combustión en solución es una tecnología alternativa a la utilizada actualmente por la industria de pigmentos inorgánicos.
\end{abstract}

Palabras clave-Colorimetría, combustión en solución, espinela, pigmento cerámico, reacción de estado sólido.

Abstract- In this work spinel structure $\mathrm{CuCr}_{2} \mathrm{O}_{4}$ was made by the alternative solution combustion method, which consists of a reaction between a fuel and an oxidizing agent. Inorganic pigment was also obtained by the method of solid state reaction, which uses a temperature of $1000{ }^{\circ} \mathrm{C}$ and a process time of $24 \mathrm{~h}$, in this case impurities of $\mathrm{Cr}_{2} \mathrm{O}_{3}$ were also presented. The crystal structure of synthesized powder was characterized by the technique of X-ray diffraction (XRD). The morphology of powders was determined by scanning electron microscopy (SEM). Finally, the spectrophotometry in the range visible (400 $\mathrm{nm}-700 \mathrm{~nm}$ ) and colorimetry CIE La*b* showed that the powders are black. It is concluded that make $\mathrm{CuCr}_{2} \mathrm{O}_{4}$ by solution combustion is more energy efficient than the traditional method, in the first case is used as process conditions a temperature of $700{ }^{\circ} \mathrm{C}$ and a time of $6 \mathrm{~h}$, which shows that the route solution combustion technology is an alternative way to the currently used in the inorganic pigments industry

Keywords - Colorimetry, solution combustion, spinel, ceramic pigment, solid state reaction.

\section{INTRODUCCIÓN}

Los pigmentos inorgánicos son materias primas que dan el color final a los productos obtenidos por la industria cerámica, los cuales se caracterizan por poseer propiedades físicas y químicas que permitan entregar productos de elevada calidad. Se define un pigmento cerámico como una sustancia inorgánica que, mezcladas en proporciones entre el $1 \%$ y el 5 $\%$ en peso con matrices vítreas o de pasta cerámica, genera la coloración uniforme de las mismas, y que debe reunir las siguiente características: ser estable a altas temperaturas, insoluble en la matriz vítrea y no modificar las propiedades de la matriz [1].

Entre las estructuras más empleadas por la industria cerámica se encuentran los que tienen la estructura espinela, siguiendo la clasificación de pigmentos cerámicos encontrada en el CPMA (Color Pigment Manufacturers Association) [2].

Entre los pigmentos inorgánicos más utilizados para dar pigmentación negra, se tiene la estructura espinela $\mathrm{CuCr}_{2} \mathrm{O}_{4}$, el cual es un semiconductor de ancho de banda limitado, y por 
tal motivo ha sido empleado como catalizador para reacciones químicas [3], pinturas [4], ánodo para baterías de ión Li [5] y pigmento cerámico [6]. El método de reacción que ha sido usado para su obtención en escala industrial es el método de reacción de estado sólido [7], el cual presenta las limitaciones antes mencionadas, además es necesario realizar una etapa posterior de molienda, debido a la aglomeración de los polvos, lo cual adiciona impurezas provenientes de los elementos moledores, que cambian la coloración de lote a lote.

Muchas técnicas de síntesis alternativa de polvos cerámicos han sido empleadas para obtener $\mathrm{CuCr}_{2} \mathrm{O}_{4}$, tales como: coprecipitación [8], descomposición térmica de oxalatos [9], y el método sonoquímico [10], entre otros. En la síntesis de polvos cerámicos, el método de combustión en solución ha sido empleado, debido a que los polvos obtenidos presentan alta pureza, bajas temperaturas de procesamiento, montaje experimental sencillo, y distribución de partícula más homogénea [11].

En la presente investigación trabajo, la síntesis del pigmento inorgánico con estructura espinela $\mathrm{CuCr}_{2} \mathrm{O}_{4}$ por el método de combustión en solución es explorado usando urea como combustible y nitratos de cromo y cobre como precursores, además se sintetiza la misma estructura por el método de reacción de estado sólido, el cual es el empleado por la industria de pigmentos cerámicos, con el objetivo de discutir las diferencias de color de los polvos obtenidos.

\section{PROCEDIMIENTO EXPERIMENTAL}

\section{A. Materiales y métodos}

\section{Síntesis por combustión en solución}

Como ya se había mencionado, el método de síntesis de combustión en solución es una ruta e síntesis química que permite obtener polvos cerámicos, cuyo montaje experimental como se observa en la figura 1 es simple, y consistió en el siguiente procedimiento, inicialmente se pesó $6.82 \mathrm{~g}$ de Urea (pureza 99\% Carlo Erba), y se adicionaron a un beaker de 500 $\mathrm{ml}$ en el cual habían $100 \mathrm{ml}$ de agua desionizada, luego, la mezcla se dejó en agitación magnética a $200 \mathrm{rpm}$ y temperatura de $25^{\circ} \mathrm{C}$ durante $10 \mathrm{~min}$, esto con el fin de homogenizar la solución acuosa, después se pesaron $13.88 \mathrm{~g}$ de nitrato de cromo nonahidratado (pureza $98 \%$ Panreac) y se adicionaron al beaker de $500 \mathrm{ml}$ de la solución inicial, a continuación, se pesó $4.18 \mathrm{~g}$ de nitrato de cobre trihidratado (99\% R-A Chemicals) y también fueron llevados a la mezcla inicial, el pH fue medido y se encontró que era de 1.7 y que no se formaban precipitados en el sistema estudiado, todas las etapas anteriores se mantuvieron a una temperatura de $25^{\circ} \mathrm{C}$, después de 20 min de agitación, se procedió a aumentar la temperatura lentamente hasta $70^{\circ} \mathrm{C}$ con el fin de evaporar el agua del sistema y formar un sistema gelificado, cuando se evaporó toda el agua, se retiró el agitador del sistema y a continuación se calentó en una plancha calefactorá a $500{ }^{\circ} \mathrm{C}$, temperatura a la que ocurrió la reacción de combustión en solución, generándose una llama que se propaga por el sistema en un tiempo de pocos segundos, finalmente se obtuvo un polvo negro, este polvo fue calcinado a $700{ }^{\circ} \mathrm{C}$ y 6 $\mathrm{h}$, con el objetivo de comparar la formación de fase esperada $\mathrm{CuCr}_{2} \mathrm{O}_{4}$.

La ecuación (1) corresponde a la reacción estequiométrica de óxido- reducción para la combustión en solución, usando urea como combustible, los cálculos fueron realizados, suponiendo que la reacción de combustión es completa.

$$
\begin{aligned}
& \mathrm{Cu}\left(\mathrm{NO}_{3}\right)_{3(l)}+2 \mathrm{Cr}\left(\mathrm{NO}_{3}\right)_{3(l)}+6.66 \mathrm{CO}\left(\mathrm{NH}_{2}\right)_{2(l)} \rightarrow \\
& \mathrm{CuCr}_{2} \mathrm{O}_{4(s)}+10.66 \mathrm{~N}_{2(g)}+6.66 \mathrm{CO}_{2(g)}+13.32 \mathrm{H}_{2} \mathrm{O}_{(g)}
\end{aligned}
$$

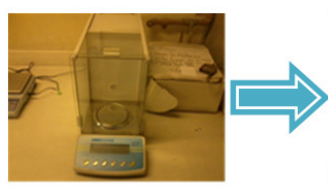

Balanza

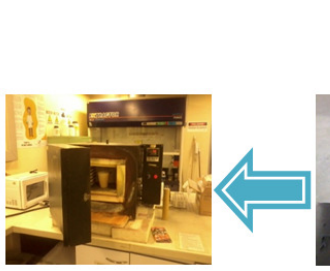

Calcinación

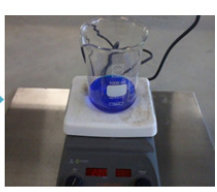

SIn de precursores

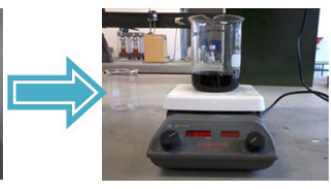

Formación del gel

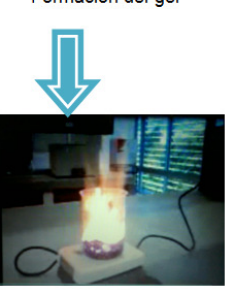

Autocombustion

Figura 1. Montaje experimental síntesis por combustión en solución.

\section{Síntesis por reacción de estado sólido}

La ruta de síntesis como se describe en la figura 2, consistió en el siguiente procedimiento. Inicialmente, se pesaron los precursores de los cationes metálicos: $6.25 \mathrm{~g}$ de óxido de cromo (III) (97\% nubiola) y $10.43 \mathrm{~g}$ de nitrato de cobre trihidratado (99\% R-A Chemicals), y fueron llevados a un mortero de porcelana donde se molturaron en seco y manualmente durante $3 \mathrm{~h}$, esto con el fin de lograr una alta homogeneidad y causar el rompimiento de los agregados de las materias primas, lo cual facilitaría la cinética de reacción química, luego, la mezcla se adicionó a un crisol de alúmina y se llevó a un horno eléctrico, el cual se programó con una rampa de calentamiento de $10{ }^{\circ} \mathrm{C} / \mathrm{min}$ desde $25^{\circ} \mathrm{C}$ hasta 1000 ${ }^{\circ} \mathrm{C}$, después, el sistema se mantuvo a esta temperatura por $6 \mathrm{~h}$, la cual es la condición de reacción química, a continuación, el sistema se enfrió hasta temperatura ambiente, esto ocurrió por inercia, ya que el horno eléctrico no tiene control de enfriamiento. Los cálculos estequiométricos se realizaron de acuerdo a las reacciones químicas balanceadas que se presentan en las ecuaciones 3 y 4, las cuales corresponden a una descomposición térmica del nitrato para producir el óxido 
de cobre (II) y después en una reacción de formación de la fase $\mathrm{CuCr}_{2} \mathrm{O}_{4}$.

Descomposición de nitrato para producir óxido cobre (II)

$$
\mathrm{Cu}\left(\mathrm{NO}_{3}\right)_{3(s)} * 3 \mathrm{H}_{2} \mathrm{O} \rightarrow \mathrm{CuO}_{(s)}+\mathrm{O}_{2(g)}+\mathrm{NO}_{2}(g)
$$

Reacción entre el óxido cobre (II) y el óxido de cromo (III)

$$
\mathrm{CuO}_{(s)}+\mathrm{Cr}_{2} \mathrm{O}_{3(s)} \rightarrow \mathrm{CuCr}_{2} \mathrm{O}_{4(s)}
$$

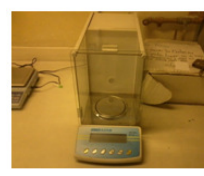

Balanza

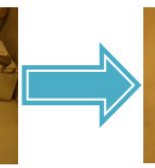

Molturación

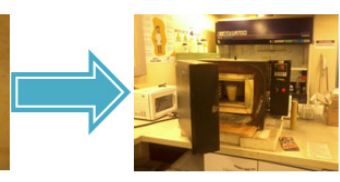

Calcinación
Figura 2. Montaje experimental síntesis por reacción de estado sólido.

Para determinar la estructura cristalina de los polvos obtenidos, se usó la técnica de difracción de rayos X (DRX). Se empleó un difractometro de doble círculo multipropósito X-Pert-Pro Panalytical, y los estudios fueron realizados mediante difractogramas de rayos $X$ en el rango desde $2 \theta=$ $17^{\circ}$ hasta $2 \theta=67^{\circ}$ en la geometría Bragg Brentano con radiación $\mathrm{Cu}-\mathrm{K}_{\alpha}(\lambda=0.15406 \mathrm{~nm})$ y paso de $0.02 \%$. Para la identificación de las fases cristalinas, los resultados experimentales fueron interpretados en el sofwaret X'Spert High Score y comparandos con la base de datos interna PFD (Powder Diffraction File).

La morfología de las superficies policristalinas fue analizada por microscopía electrónica de barrido (MEB) usando un microscopio electrónico SEM-EDX JEOL JSM 5910LV detectores BES (electrones retroproyectados), con un voltaje de $12 \mathrm{kV}$ para la generación de imágenes y a una distancia de trabajo de $10 \mathrm{~mm}$.

Los análisis de espectrofotometría visible y las coordenadas colorimétricas CIELa*b* 1976, fueron realizados en un espectrofotómetro UV-VIS, marca Glacier ${ }^{\mathrm{TM}} \mathrm{X}$ con rango espectral de detección entre 200 y 1050 nm, se utilizó geometría de medida de $45^{\circ}: 0^{\circ}$, iluminante D65 y observador estándar CIE 1964 de $10^{\circ}$, empleando la norma ASTM E30813 [12].

\section{RESULTADOS Y ANÁLISIS}

En la figura 3, se muestran los difractogramas de rayos $\mathrm{X}$ para la estructura $\mathrm{CuCr}_{2} \mathrm{O}_{4}$ sintetizada por el método de combustión en solución, es importante resaltar que los picos del polvo obtenido inmediatamente termina la combustión a $500{ }^{\circ} \mathrm{C}$ no indexan con los picos del patrón para $\mathrm{CuCr}_{2} \mathrm{O}_{4}$ con PDF 034-0424, el cual se caracteriza por presentar un pico característico a $2 \theta=18,606^{\circ}$, además se puede apreciar que dicho polvo es una mezcla entre el óxido de cromo $\mathrm{Cr}_{2} \mathrm{O}_{3}$ con PDF 038-1479 y el óxido de cobre CuO con PDF 005-0661, la explicación de esta situación se debe a que la temperatura alcanzada durante la reacción de combustión empleando la urea combustible no es lo suficientemente alta para obtener la estructura esperada, por tal motivo se realizó una calcinación a $700{ }^{\circ} \mathrm{C}$ y $6 \mathrm{~h}$, la cual mostro la obtención de la fase pura $\mathrm{CuCr}_{2} \mathrm{O}_{4}$, a realizar la comparación de los picos de difracción con el estándar y verificar la presencia del pico característico mencionado, dicha estructura cristaliza en el sistema cristalino cúbico tetragonal, con grupo espacial I-42d, y además se observa la desaparición de los picos característicos del óxido de cromo a $2 \theta=24^{\circ}$ y del óxido de cobre a $2 \theta=49^{\circ}$, aproximadamente.

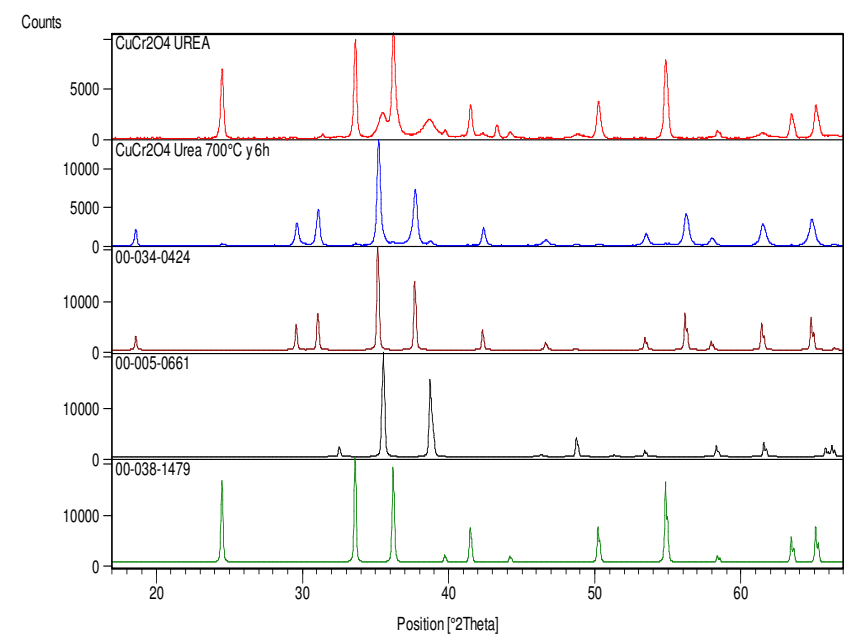

Figura 3. Difractogramas de rayos $\mathrm{X}$ para polvo obtenido por combustión en solución.

El difractograma del polvo de la muestra obtenida por reacción de estado sólido es mostrado en la figura 4, se puede observar que existe el pico característico de la $\mathrm{CuCr}_{2} \mathrm{O}_{4}$ a $2 \theta=$ $18,606^{\circ}$ del patrón PDF 034-424, pero también se encuentran picos característicos del $\mathrm{Cr}_{2} \mathrm{O}_{3}$ a $2 \theta=25^{\circ}$ y $2 \theta=33.5^{\circ}$ del patrón PDF 038-1479, y el $2 \theta=29^{\circ}$, correspondiente al $\mathrm{CuO}$ del patrón PDF 005-0661, por tal motivo las condiciones empleadas en el proceso no son suficientes para obtener las fase pura $\mathrm{CuCr}_{2} \mathrm{O}_{4}$, se tendría que aumentar la temperatura del proceso con el fin de aumentar la velocidad de reacción, ya que en el caso del método de reacción de estado sólido, la difusión de las especies iónicas es baja, otra manera de aumentar el rendimiento de obtención sería aumentar el tiempo de residencia en el horno a la temperatura de $1000^{\circ} \mathrm{C}$, aumentando el tiempo de contacto entre los reactantes, con el fin de permitir que las especies se puedan difundir, o emplear fundentes, los cuales generarían una fase líquida, disminuyendo la viscosidad del sistema y por tal motivo se aumentaría la cinética de reacción química. 


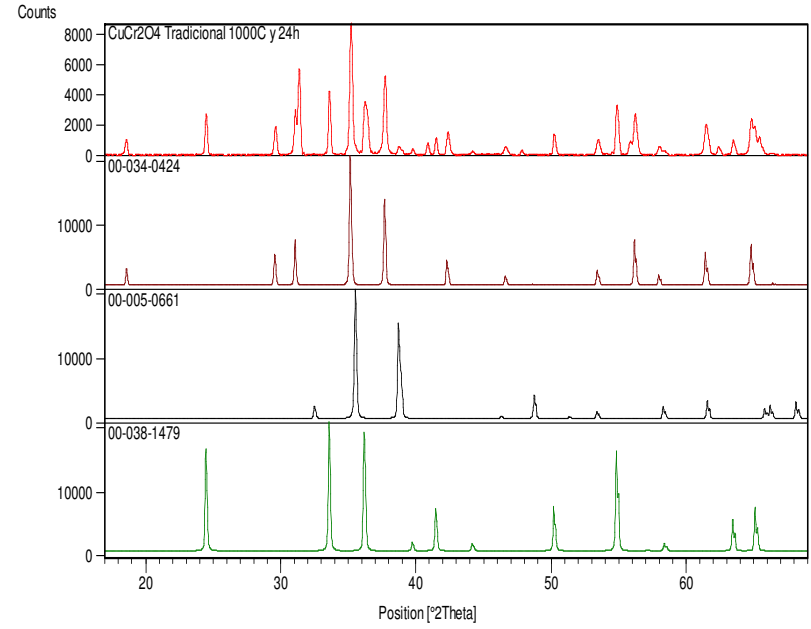

Figura 4. Difractograma de rayos X para polvo obtenido por reacción de estado sólido.

Las imágenes de SEM que se muestran en la figura 5, permiten observar que en ambas rutas de síntesis se obtienen aglomerados de partículas menores a $5 \mu \mathrm{m}$, pero en el caso de la síntesis de combustión en solución la aglomeración es menor que en la síntesis de estado sólido, esto se debe a que en la reacción en estado sólido, se emplea la temperatura de $1000{ }^{\circ} \mathrm{C}$ y un tiempo de $24 \mathrm{~h}$ de reacción, lo que permite que varias partículas se puedan unir para formar dichas aglomeraciones, mientras que en el caso de la síntesis por combustión se tienen condiciones de proceso menores, además no se encuentra una morfología definida en ambos casos, para la imagen correspondiente a la síntesis por estado sólido hay zonas que muestran partículas con geometría octaédrica, en el caso de la síntesis por combustión en solución también se aprecia esta morfología.
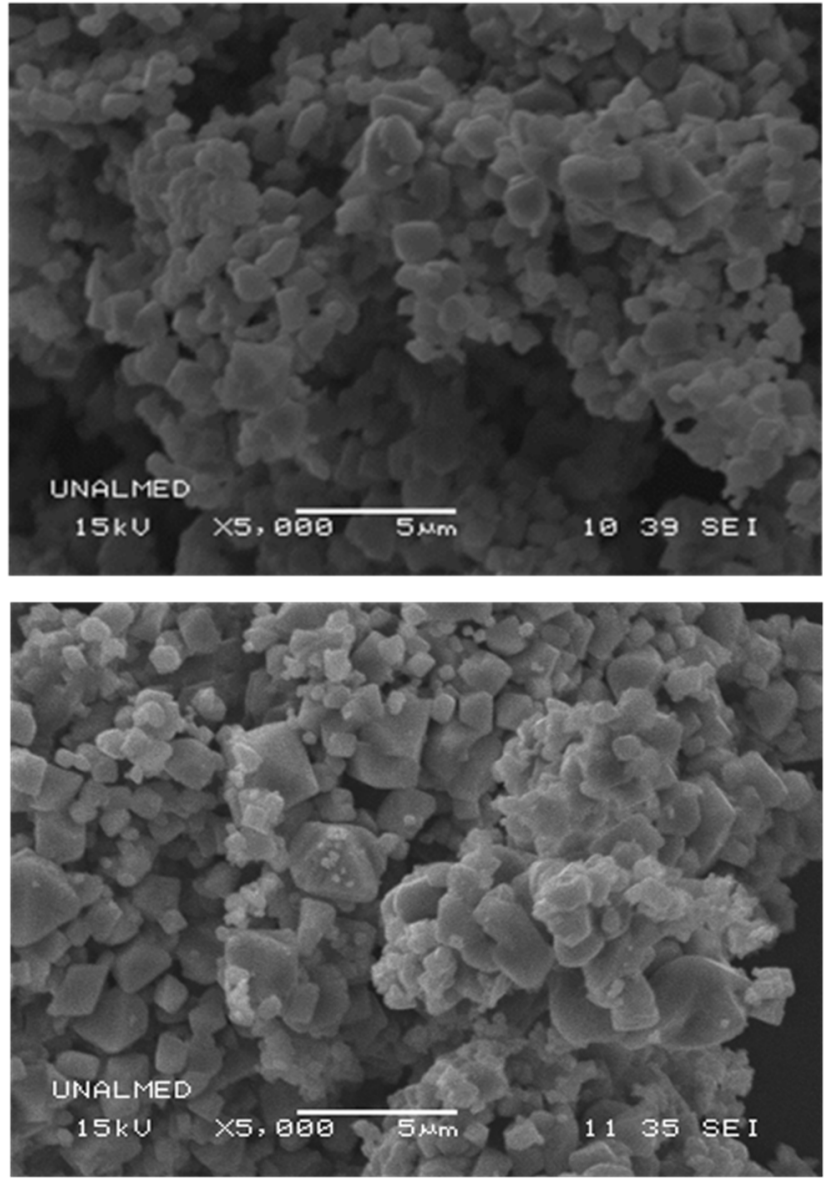

Figura 5. Micrografías de microscopía electrónica de barrido, imagen superior: Combustión en solución, imagen inferior: reacción de estado sólido.

Los espectros de reflectancia difusa en la región visible de la figura 6, muestran porcentajes de factor de reflectancia difusa menores al $10 \%$ para los dos polvos obtenidos por ambas rutas de síntesis, pero en el caso del polvo obtenido por el método de reacción de estado sólido se observa una banda con un pico aproximadamente a $540 \mathrm{~nm}$, el corresponde a la región verde del espectro electromagnético, y que está de acuerdo con la fase de $\mathrm{Cr}_{2} \mathrm{O}_{3}$, la cual se observa en el DRX. En el caso del espectro de reflectancia difusa para el polvo sintetizado por el método de combustión en solución este pico no se encuentra, que de nuevo corresponde a lo observado por DRX para dicho polvo. 


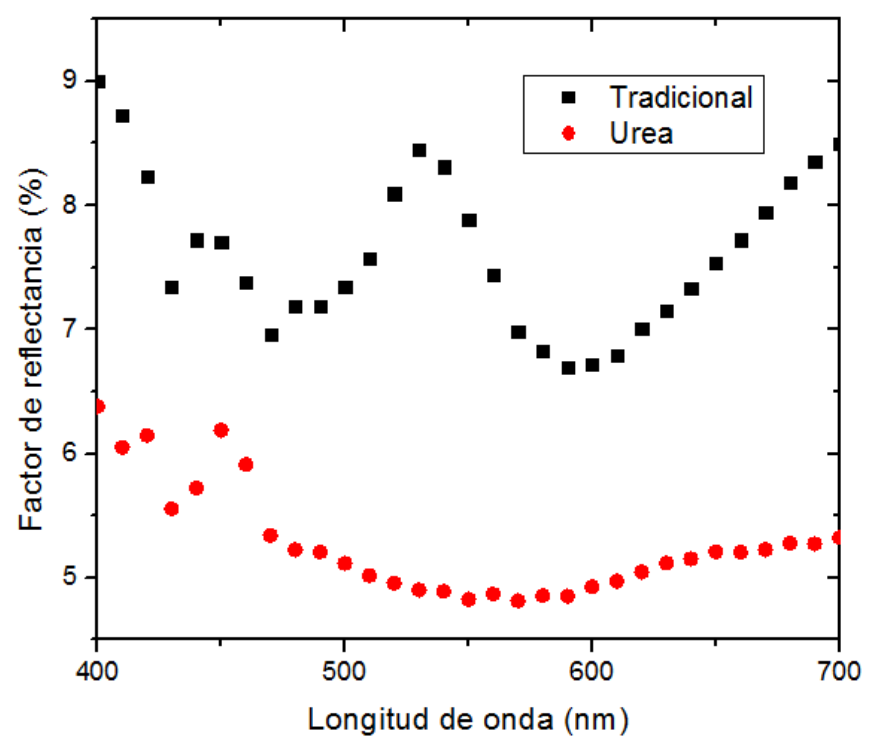

Figura 6. Espectro de reflectancia difusa visible para los polvos obtenidos.

En la tabla 1 se muestran las coordenadas colorimétricas de los polvos obtenidos por las rutas de síntesis empleadas en esta investigación, en el sistema de color CIEL*a*b*, la coordenada $\mathrm{L}^{*}$ expresa luminosidad, para valores de $\mathrm{L}^{*}$ cercanos a 100 se dice que la muestra es de alta claridad, y en el caso de valores de $\mathrm{L}^{*}$ cercanos a 0 la muestra posee baja claridad, de acuerdo con lo anterior las muestras poseen una claridad baja, siendo más claro el polvo obtenido por el método de reacción de estado sólido, que el obtenido por combustión en solución, lo cual se explica por la presencia de la fase secundaria $\mathrm{Cr}_{2} \mathrm{O}_{3}$ en la muestra, que muestra una banda más intensa en la región verde. Para las coordenadas a*, valores positivos significan que la muestra es roja y valores negativos que es verde, siendo cero el punto acromático, en el caso de las dos muestras se puede apreciar que sus coordenadas $\mathrm{a}^{*} \mathrm{y} \mathrm{b}^{*}$ se acercan al eje acromático, y de nuevo hay un valor negativo para la coordenada $a^{*}$ del método de reacción de estado sólido.

\begin{tabular}{|c|c|c|c|c|}
\hline Método de síntesis & $\mathrm{L}^{*}$ & $\mathrm{a}^{*}$ & $\mathrm{~b}^{*}$ & $\mathrm{C}^{*}$ \\
\hline Tradicional & 32.818 & -1.956 & -0.614 & 2.050 \\
\hline $\begin{array}{c}\text { Combustión en } \\
\text { solución }\end{array}$ & 26.666 & 1.438 & -3.433 & 3.722 \\
\hline
\end{tabular}

Tabla 1. Coordenadas colorimétricas CIEL*a*b* de los polvos obtenidos.

Con el fin de calcular la diferencia de cromaticidad entre ambas mezclas se realizó una transformación del espacio de color CIEL*a*b* al CIEL*C*h*, con el objetivo de calcular la diferencia de color entre las dos muestras, siguiendo la norma ASTM D2244-15 [13], donde $C^{*}$ es el croma o saturación de la muestra y es definido como la pureza del color, $\mathrm{y} \mathrm{h}^{*}$ es el tono de la muestra y es definido, las ecuaciones utilizadas en los cálculos se resumen en las ecuaciones 4 hasta 9 .

$$
\begin{aligned}
& \Delta E_{a b}=\left[\left(\Delta L_{a b}^{*}\right)^{2}+\left(\Delta C_{a b}^{*}\right)^{2}+\left(\Delta H^{*}{ }_{a b}\right)^{2}\right]^{0.5} \\
& C_{a b}^{*}=\sqrt{a^{* 2}+b^{* 2}}(5) \\
& \Delta C^{*}{ }_{a b}=\left(C_{a b}^{*}\right)_{1}-\left(C_{a b}^{*}\right)_{2} \quad(6) \\
& \Delta L^{*}{ }_{a b}=\left(L_{a b}^{*}\right)_{1}-\left(L_{a b}^{*}\right)_{2}(7) \\
& \Delta H_{a b}^{*}=s\left[2\left(C_{a b_{1}}^{*} C_{a b_{2}}^{*}-a_{1}^{*} a_{2}^{*}-b_{1}^{*} b_{2}^{*}\right)\right]^{0.5}(8)
\end{aligned}
$$

Si: $a_{2}^{*} b_{1}^{*}>a_{1}^{*} b_{2}^{*}(9)$, por lo tanto $\mathrm{s}=1$, en caso contrario $\mathrm{s}=-1$

Para los polvos sintetizados se define:

1: Tradicional

2: Combustión en Solución

Empleando la ecuación 9, se tiene:

$(1.438)(-0.614)>(-1.956)(3.722)$

$-0.8829>-7.2802$

Por lo tanto, $\mathrm{s}=1$.

De las ecuaciones 6 hasta 8 , se obtiene: $\Delta \mathrm{C}^{*}{ }_{\mathrm{ab}}=-1.672, \Delta \mathrm{H}^{*}{ }_{\mathrm{ab}}$ $=5.153, \Delta \mathrm{L}_{\mathrm{ab}}^{*}=6.152$ y $\Delta \mathrm{E}_{\mathrm{ab}}=8.197$.

De acuerdo con la norma ASTM D2244-15, el ojo humano es capaz de diferenciar dos muestras de color como diferentes, si la diferencia de color es mayor que $1(\Delta \mathrm{E}>1)$, lo cual significa que en el caso de las muestras de esta investigación son cromáticamente diferentes, lo cual se debe a la presencia de $\mathrm{Cr}_{2} \mathrm{O}_{3}$ en el polvo obtenido por el método de reacción de estado sólido, y que también fue confirmado por la presencia de una banda de reflectancia con un pico máximo en $540 \mathrm{~nm}$. Por lo tanto, para obtener una diferencia de color menor se debe preparar el polvo por reacción de estado sólido a unas condiciones de temperatura mayores a $100{ }^{\circ} \mathrm{C}$ o a tiempos de residencia en el horno mayores a $6 \mathrm{~h}$, de esta manera se puede verificar la importancia de obtener la estructura pura $\mathrm{CuCr}_{2} \mathrm{O}_{4}$ por el método de combustión en solución a $600{ }^{\circ} \mathrm{C}$ y $6 \mathrm{~h}$, lo cual se convierte en una tecnología alterna energéticamente más eficiente.

\section{CONCLUSIONES}

Se obtuvo el pigmento inorgánico con la estructura $\mathrm{CuCr}_{2} \mathrm{O}_{4}$, por el método de combustión en solución usando como combustible la urea. Las condiciones del proceso fueron de $700{ }^{\circ} \mathrm{C}$ y $6 \mathrm{~h}$, las cuales son menores a las utilizadas por el método de reacción de estado sólido, que para efectos comparativos se sintetizó a una temperatura y tiempo de 
reacción de $1000{ }^{\circ} \mathrm{C}$ y $24 \mathrm{~h}$, respectivamente. Dicho ahorro energético podría ser importante para la industria de pigmentos inorgánicos.

Se determinó la importancia de obtener fases puras, para realizar comparaciones colorimétricas que permitan determinar diferencias de color aceptables por la industria, con el fin de obtener una metodología apropiada de control de calidad de los productos manufacturados.

\section{AGRADECIMIENTOS}

Los autores expresan sus agradecimientos al laboratorio de Cerámicos y Vítreos, y al laboratorio de Química del Cemento de la Universidad Nacional de Colombia sede Medellín, por la disponibilidad de sus equipos para la realización de esta investigación.

\section{REFERENCIAS}

[1]. G. Monros, El color de la cerámica: nuevos mecanismos en pigmentos para los nuevos procesados en la industria cerámica, vol I. Castellón: Publications de la Universitat Jaume I, 2003, p. 17.

[2]. CPMA Classification of Chemical Descriptions and Usage of the Complex Inorganic Color Pigments. [Online]. Available: http://www.pigments.org/cms/publications/

[3]. S. S. Acharyya, S. Ghosh, S. Adak, D. Tripathi, and R. Bal, "Fabrication of $\mathrm{CuCr} 2 \mathrm{O} 4$ spinel nanoparticles: A potential catalyst for the selective oxidation of cycloalkanes via activation of Csp3-H bond," Catal Commun, vol. 59, pp. 145-150, Ene. 2015. Doi: 10.1016/j.catcom.2014.10.015

[4]. Q. Geng, X.Zhao, X. Gao, S Yang, and G. Liu, "Low temperatura combustion synthesis of $\mathrm{CuCr}_{2} \mathrm{O}_{4}$ spinel podwer for spectrally selective paints," J Sol-Gel Sci Tech, vol. 61, pp. 281-288, Ene. 2012. Doi: 10.1007/s10971-011-2625-2

[5]. M. Lao, J. Shu, L. Shao, X. Lin, X, K. Wu, K, M. Shui, and Y. Ren, "Enhanced electrochemical performance of ag-coated $\mathrm{CuCr}_{2} \mathrm{O}_{4}$ as anode material for lithium-ion batteries," Ceram Int, vol. 40, pp. 11899-11904, Sep. $2014 . \quad$ Doi: 10.1016/j.ceramint.2014.04.025

[6]. W. Wang, X. Zheng, P. Li, H. Xu, and Y. Zhang, "Synthesis of ultrafine copper chromite black pigment by chemical coprecipitation using SBDSassisted urea," Huagong Xuebao/CIESC Journal, vol. 64, pp. 1847-1854, May. 2013. Doi: 10.3969/j.issn.0438-1157.2013.05.047

[7]. R. Eppler, "Inverse spinel pigments," J. Am .Ceram .Soc, vol. 66, pp. 791-801. 1983.

[8]. Z. Hu, Y. Qin, H. Zhou, J. Kang, S. Zhai, and H. Gao, "Preparation and photoelectric properties of CuCr2O4 nanopowders," Adv Mat Res, vol. 284-286, pp. 974-979. Jul. 2011.
Doi: 10.4028/www.scientific.net/AMR.284-286.974

[9]. R. Prasad, "Highly active copper chromite catalyst produced by thermal decomposition of ammoniac copper oxalate chromate," Mater Lett, vol. 59(2930), pp. 3945-3949. Dic. 2005. Doi: 10.1016/j.matlet.2005.07.041

[10]. M. Shahidzadeh, P. Shabihi, and S. M. Pourmortazavi, "Sonochemical preparation of copper(II) chromite nanocatalysts and particle size optimization via taguchi method," J Inorg Organomet P, Feb. 2015. En impresión.

[11]. K.C. Patil, M. S. Hedge, T. Rattan, and S.T. Aruna, Chemistry of Nanocrystalline Oxide Materials Combustion Synthesis, Properties and Applications, vol. 1. Singapore: World Scientific Publishing Co. Pte. Ltd, 2008, p. 5.

[12]. Standard Practice for Computing the Colors of Objects by Using the CIE System, ASTM Standard E308-13, Nov. 2013.

[13]. Standard Practice for Calculation of Color Tolerances and Color Differences from Instrumentally Measured Color Coordinates, ASTM Standard D2244-15, Ene. 2015. 